\title{
Médiévales
}

Langues, Textes, Histoire

67 | automne 2014

Histoires de Bohême

\section{Claude GAUVARD, Alessandro stella (dir.), Couples en} justice, IV ${ }^{e}-X I X^{e}$ siècle

Publications de la Sorbonne (« Homme et société », 45), 2013, 250 p.

\section{Emmanuelle Santinelli}

\section{(2) OpenEdition}

1 Journals

\section{Édition électronique}

URL : https://journals.openedition.org/medievales/7358

DOI : $10.4000 /$ medievales. 7358

ISSN : $1777-5892$

\section{Éditeur}

Presses universitaires de Vincennes

\section{Édition imprimée}

Date de publication : 31 décembre 2014

Pagination : 205-207

ISBN : 978-2-84292-422-5

ISSN : 0751-2708

\section{Référence électronique}

Emmanuelle Santinelli, "Claude gauvard, Alessandro stella (dir.), Couples en justice, $v^{e}-x i x^{e}$ siècle ", Médiévales [En ligne], 67 | automne 2014, mis en ligne le 09 mars 2015, consulté le 22 avril 2022. URL: http://journals.openedition.org/medievales/7358; DOI : https://doi.org/10.4000/medievales.7358

Ce document a été généré automatiquement le 22 avril 2022

Tous droits réservés 


\title{
Claude GAUVARD, Alessandro STELLA (dir.), Couples en justice, IV ${ }^{e}$-XIX $X^{e}$ siècle
}

Publications de la Sorbonne («Homme et société », 45), 2013, 250 p.

\author{
Emmanuelle Santinelli
}

\section{RÉFÉRENCE}

Claude GAUVARD, Alessandro STELla (dir.), Couples en justice, IV $V^{e} X I X^{e}$ siècle, Paris,

Publications de la Sorbonne («Homme et société », 45), 2013, 250 p.

1 Au croisement des recherches sur le mariage, la famille, les femmes, le genre, la violence et la sexualité, ce livre - qui constitue les actes du colloque tenu à Paris en octobre 2010 - analyse, dans un cadre chronologique, spatial et social large, une pratique qui, banale aujourd'hui dans le monde occidental, n'en a pas moins une existence ancienne, sans pour autant avoir retenu, jusqu'à récemment, l'attention des historiens. L'objectif est donc de saisir, dans leurs diversités, les raisons, les acteurs et les conséquences du recours à la justice (quelle qu'en soit la forme) par les couples (hétérosexuels, puisqu'alors seuls reconnus) et ceux qui les soutiennent ou les contraignent, ce qui marque le passage du conflit de la sphère du privé à celle du public.

2 L'ouvrage propose, après l'introduction synthétique d'Alessandro Stella (cadre, problématique, plan), onze articles rédigés par des historiens européens, spécialistes des questions familiales et/ou de justice, ce dont témoignent les notices relatives aux auteurs en fin de volume (p. 245-248). Les articles sont organisés en trois parties qui traitent du recours à une forme ou une autre de justice du fait d'abord du rapt (I), ensuite de la normalisation du couple (II), ainsi que, pour finir, des stratégies du couple et des autres acteurs qui interviennent dans ce cadre (III). La conclusion de claude Gauvard souligne l'homogénéité observée, malgré la diversité des cas étudiés, quant aux réactions, au déroulement des affaires, à la perception de la part d'initiative des uns et des autres, ou à l'importance de l'honneur, avant de dégager trois grandes pistes 
de réflexion, ce qui confère une certaine unité à l'ensemble. Homogénéité ne signifie néanmoins pas uniformité: les cas envisagés témoignent aussi des différences régionales et des évolutions entre la fin de l'Empire romain et l'époque industrielle. Les index des noms de personnes et de lieux (on regrettera du coup l'absence d'un index rerum), ainsi que le résumé des articles en français et en anglais, en font un outil de travail pratique et indispensable pour tous ceux qui travaillent, non seulement sur les structures familiales et la justice, mais aussi sur les rapports entre les sexes ou encore les conflits.

3 La première partie, consacrée à la question du rapt, est diachronique : la pratique est successivement analysée au haut Moyen Âge (par Sylvie Joye), dans la Flandre et le Brabant des derniers siècles médiévaux (par Myriam Greilsammer) et dans la Roumanie du XIX siècle (par Nicoleta Roman). Même si les contextes sont différents, les trois études montrent que les affaires de rapt concernent, plus que le couple, leurs familles (dont les droits, les biens et l'autorité sont remis en cause) et les autorités publiques (responsables de l'ordre, voire bénéficiaires des sanctions prises contre les acteurs du rapt), qu'elles soient centrales ou locales, religieuses ou laïques, etc. Dans ces conditions, le fait que la femme est consentante ou non importe peu. Dans tous les cas, il faut se méfier de la documentation qui peut, selon la finalité recherchée, soit présenter les faits selon un récit acceptable contribuant à la reconnaissance du couple, soit insister sur la violence de l'acte (qu'elle soit réelle ou inventée) pour obtenir et justifier sa condamnation. En outre, il y a souvent décalage entre la condamnation sévère des lois et la pratique qui témoigne de compromis négociés à l'amiable entre les familles : il en résulte finalement un petit nombre seulement de cas de rapt traités en justice, parmi lesquels figurent souvent ceux qui ont échoué. Il n'en reste pas moins que certaines évolutions sont perceptibles, ce qui ne signifie pas qu'elles sont linéaires, notamment quant à l'intervention des autorités centrales (incertaine au haut Moyen Âge, accrue à la fin du Moyen Âge, en retrait au XIX siècle) et à la part d'initiative de la femme (plus importante à la fin du Moyen Âge et surtout au xix siècle qu'au haut Moyen Âge).

4 La seconde partie, centrée sur la normalisation du couple, envisage, pour la fin du Moyen Âge et l'époque moderne, les relations entre la cellule conjugale et le monde judiciaire: soit que la première se tourne vers le second pour régler le conflit qui l'agite, soit que le tribunal assigne le couple à comparaitre pour non-respect des normes fixées. Les tribunaux sont souvent religieux : catholiques, comme dans le NordOuest de la France (analysé par Carole Avignon), ou dans les réponses de la sacrée congrégation constituée à l'issue du concile Trente pour répondre aux questions pratiques posées par les évêques (étudiées par Gabriella Zarri), mais aussi juifs (cas traité par Roni Weinstein). Cela n'exclut cependant pas l'intervention ou le recours à la justice laïque, voire à l'infrajudiciaire (dont la complémentarité et la concurrence dans les États italiens retiennent plus particulièrement l'attention de Daniela Lombardi). Les cas de recours aux tribunaux, quels qu'ils soient, sont d'une grande diversité : du fait de mariages considérés comme clandestins, avec des situations variées (Carole Avignon); du fait de mariages présumés, de promesses de mariage non tenues ou de séduction (Daniela Lombardi) ; du fait du non-respect des conditions qui font la validité du lien matrimonial (Gabriella Zarri) ou des comportements conformes à la loi religieuse et à la morale sociale (Roni Weinstein). Malgré la diversité des approches et des cadres (chronologiques et géographiques) envisagés, les quatre études témoignent de la 
volonté des autorités (quelles qu'elles soient) de contrôler les affaires familiales, et plus particulièrement le mariage et les relations conjugales, qu'il s'agit plus souvent de conforter ou de légitimer que de dissoudre. Si le tribunal est une institution de contrainte, il peut aussi être un outil de normalisation conjugale, instrumentalisé plus ou moins consciemment par les couples, voire leurs proches. La volonté d'encadrer le couple, et plus généralement la famille, n'exclut pas des positions divergentes au sein des autorités, une évolution des interprétations et donc de la législation, des résistances parmi les populations à respecter les comportements imposés et, dans la pratique, une certaine adaptation de la loi aux réalités sociales. Les quatre articles soulignent aussi qu'en marge des tribunaux, plus sollicités qu'auparavant, entre en ligne de compte la pression sociale, imbriquée de façon inextricable dans le système juridique : soit que celle-ci influe sur la procédure juridique, soit que le recours à la justice soit utilisé pour obtenir un compromis à l'amiable. Selon le type de situations conflictuelles, hommes et femmes agissent tantôt de concert, tantôt séparément, avec dans ce cas des motivations et des stratégies qui diffèrent souvent.

La troisième partie, organisée quant à elle autour des stratégies du couple face à la justice, entre le $\mathrm{xv}^{\mathrm{e}}$ et le début du $\mathrm{xIX}^{\mathrm{e}}$ siècle, s'intéresse aux types de tribunaux auxquels recourent les conjoints, solidairement ou séparément, ou qui interviennent à l'initiative ou non des justiciables, aux discours construits à leur intention, avec leurs déclinaisons masculines et féminines, au rôle joué par tous ceux qui interviennent dans la procédure (juges, témoins, prêtres, amis, voisins, parents), mais aussi à la façon dont le couple peut devenir un enjeu entre les juridictions en compétition ou un moyen pour la justice de mettre en scène son autorité. Les quatre contributions proposent, à partir de l'analyse d'affaires concrètes, des angles d'observation divers : nomadisme judiciaire (Martine Charageat), procès en séparation (Claire Chatelain), intimité et sentiments dévoilés aux juges (Alessandro Stella), usage du corps et des mots dans les procédures judiciaires (Constanţa Vintilă-Ghị̧ulescu). Les cadres d'analyse sont aussi variés: Aragon des $\mathrm{XV}^{\mathrm{e}}-\mathrm{XVI}^{\mathrm{e}}$ siècles (Martine Charageat), milieu de la haute robe parisienne du début du $\mathrm{xVIII}^{\mathrm{e}}$ siècle (Claire Chatelain), Cadix au xvIII ${ }^{\mathrm{e}}$ siècle (Alessandro Stella), Roumanie des années 1750-1830 (ConstanŢa Vintilă-GhiŢulescu). Il n'en ressort pas moins certaines convergences : les couples de toutes les couches sociales savent jouer des procédures et des juridictions et n'hésitent pas à s'en servir pour obtenir gain de cause; le recours ou le passage en justice apparaît à bien des égards comme un spectacle, avec des acteurs qui connaissent leur rôle et, manipulant paroles et corps, usent d'une argumentation prise dans une fiction théâtralisée, avec des juges qui, aidés par l'infrajudiciaire, recherchent le compromis plus que la sanction pour rétablir l'ordre, et avec le public qui exerce une sorte de ministère qui juge des réputations ; s'il n'est pas aisé de saisir ce qui est vrai dans ces mises en scène, ni les motivations et finalités réelles de chacun, il est possible d'appréhender quelques aspects du discours sur le couple, la complexité des sentiments, les relations attendues entre les conjoints, leur intégration dans les groupes familiaux et les réseaux sociaux. 